\title{
Distributed algorithms for edge dominating sets
}

\section{Suomela, Jukka}

ACM

2010

Suomela , J 2010 , Distributed algorithms for edge dominating sets . in A Richa \& R pÿGuerraoui (eds), PODC 10 : Proceedings of the 2010 ACM Symposium on Principles of pÿDistributed Computing . ACM , pp. 365374 , ACM SIGACT-SIGOPS Symposium on Principles of Distributed Computing (PODC) , Zurich , Switzerland , 25/07/2010 . https://doi.org/10.1145/1835698.18

http://hdl.handle.net/10138/27921

https://doi.org/10.1145/1835698.1835783

acceptedVersion

Downloaded from Helda, University of Helsinki institutional repository.

This is an electronic reprint of the original article.

This reprint may differ from the original in pagination and typographic detail.

Please cite the original version. 


\title{
Distributed Algorithms for Edge Dominating Sets
}

\author{
Jukka Suomela \\ Helsinki Institute for Information Technology HIIT, University of Helsinki \\ P.O. Box 68, Fl-00014 University of Helsinki, Finland \\ jukka.suomela@cs.helsinki.fi
}

\begin{abstract}
An edge dominating set for a graph $\mathcal{G}$ is a set $D$ of edges such that each edge of $\mathcal{G}$ is in $D$ or adjacent to at least one edge in $D$. This work studies deterministic distributed approximation algorithms for finding minimum-size edge dominating sets. The focus is on anonymous port-numbered networks: there are no unique identifiers, but a node of degree $d$ can refer to its neighbours by integers $1,2, \ldots, d$. The present work shows that in the port-numbering model, edge dominating sets can be approximated as follows: in $d$-regular graphs, to within $4-6 /(d+1)$ for an odd $d$ and to within $4-2 / d$ for an even $d$; and in graphs with maximum degree $\Delta$, to within $4-2 /(\Delta-1)$ for an odd $\Delta$ and to within $4-2 / \Delta$ for an even $\Delta$. These approximation ratios are tight for all values of $d$ and $\Delta$ : there are matching lower bounds.
\end{abstract}

\section{Categories and Subject Descriptors}

C.2.4 [Computer-Communication Networks]: Distributed Systems; F.2.2 [Analysis of Algorithms and Problem Complexity]: Nonnumerical Algorithms and Problems - computations on discrete structures

\section{General Terms}

Algorithms, Theory

\section{INTRODUCTION}

This work studies the approximability of the edge dominating set problem from the perspective of deterministic (non-randomised) distributed algorithms in anonymous portnumbered networks.

\subsection{Edge Dominating Sets and Matchings}

Let $\mathcal{G}$ be a simple, undirected graph with the edge set $E_{\mathcal{G}}$. A set $D \subseteq E_{\mathcal{G}}$ of edges is an edge dominating set for $\mathcal{G}$ if each edge $e \in E_{\mathcal{G}} \backslash D$ is adjacent to at least one edge in $D$. See Figure 1 for examples.

(C) ACM, 2010. This is the author's version of the work. It is posted here by permission of ACM for your personal use. Not for redistribution. The definitive version was published in Proc. 29th ACM SIGACT-SIGOPS Symposium on Principles of Distributed Computing (PODC'10, July 25-28, 2010, Zurich, Switzerland).

http://doi.acm.org/10.1145/1835698.1835783
By definition, any maximal matching is an edge dominating set. An edge dominating set is not necessarily a matching; however, given an edge dominating set $D$, it is straightforward to construct a maximal matching with at most $|D|$ edges [25]. Hence a minimum maximal matching (a maximal matching with the smallest possible number of edges) is also a minimum edge dominating set.

This is a corollary of a more general result due to Allan and Laskar [1]: if a graph is claw-free (no induced subgraph $K_{1,3}$ ), then a minimum maximal independent set is also a minimum dominating set. The line graph $L(\mathcal{G})$ of any graph $\mathcal{G}$ is claw-free, the dominating sets of $L(\mathcal{G})$ correspond to the edge dominating sets of $\mathcal{G}$, and the maximal independent sets of $L(\mathcal{G})$ correspond to the maximal matchings of $\mathcal{G}$.

\subsection{Centralised Polynomial-Time Algorithms}

From the perspective of centralised polynomial-time algorithms, the problem of finding a minimum edge dominating set is equivalent to the problem of finding a minimum maximal matching. Both of these are NP-hard optimisation problems [25], and they are hard to approximate to within factor $7 / 6-\epsilon[9]$. The problem of finding a minimum-weight edge cover is as hard to approximate as minimum-weight vertex cover [8].

The connection between matchings and edge dominating sets implies a simple 2-approximation algorithm: any maximal matching is a 2-approximation of a minimum edge dominating set. Approximating minimum-weight edge dominating sets is less straightforward, but Fujito and Nagamochi [12] show how to find a 2-approximation. Polynomialtime approximation schemes are known for planar graphs [6] and civilised graphs [15].

\subsection{Distributed Algorithms}

Edge dominating sets have received little attention in the distributed computing community. However, some results related to matchings and independent sets have straightfor-

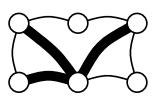

(a)

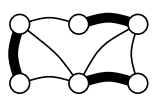

(b)

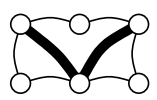

(c)

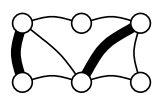

(d)
Figure 1: (a) An edge dominating set. (b) A maximal matching and hence another edge dominating set. (c) A minimum edge dominating set. (d) A minimum maximal matching and hence another minimum edge dominating set. 
ward corollaries that concern the distributed approximability of edge dominating sets.

On the positive side, one can again take any algorithm that finds a maximal matching and apply it to find a 2approximation of a minimum edge dominating set. For example, if we have unique node identifiers in the network, we can use the deterministic algorithms by Hańckowiak et al. [14] and Panconesi and Rizzi [19], with running times $O\left(\log ^{4} n\right)$ and $O\left(\Delta+\log ^{*} n\right)$ communication rounds, respectively; here $n$ is the number of nodes and $\Delta$ is the maximum degree.

The running times of these algorithms depend on $n$, and this is unavoidable if we want to achieve an approximation factor better than 3. Czygrinow et al. [10] and Lenzen and Wattenhofer [17] show that finding a constant-factor approximation of a maximum independent set in a cycle requires $\Omega\left(\log ^{*} n\right)$ communication rounds, and a simple local reduction [22] gives the same lower bound for finding a factor $3-\epsilon$ approximation of a minimum edge dominating set.

\subsection{Algorithms in Port-Numbered Networks}

The above results deal with deterministic distributed algorithms in networks with unique node identifiers. This work studies a strictly weaker model of computation: deterministic distributed algorithms in anonymous networks in the port-numbering model: there are no node identifiers, but a node of degree $d$ can refer to its neighbours by integers $1,2, \ldots, d$. See Section 2 for a formal definition of the model.

Computation in synchronous port-numbered networks has been studied for decades; one of the pioneers was Angluin [2] in 1980. However, the main focus has been on global problems such as leader election [2, 24], construction of spanning trees [24], computation of functions that depend on all nodes $[5,7,23]$, recognition of topological properties [2, 24], and graph exploration and rendezvous [16]. Such problems typically require $\Omega(n)$ communication rounds - or, in many cases, are unsolvable in the port-numbering model.

Much less is known about graph problems that are of a more local nature and have potential for efficient, highly scalable distributed algorithms. Classical packing problems such as matchings and independent sets are typically unsolvable for trivial reasons, but covering problems are more promising. Node-based covering problems (the task is to choose a subset of nodes that "covers" the graph) have been studied in prior work: for example, the vertex cover problem can be approximated within factor 2 in the port-numbering model in bounded-degree graphs [3, 4], and this approximation guarantee is tight. However, it seems that edge-based covering problems have not been studied previously in this model.

\subsection{Contributions}

The contributions are summarised in Table 1. This work presents a complete characterisation of the deterministic approximability of edge dominating sets in the port-numbering model, both in graphs of maximum degree $\Delta$ and in $d$ regular graphs, for all values of the parameters $\Delta$ and $d$. All approximation ratios are tight: there are exactly matching upper and lower bounds.

On a more conceptual level, the contributions are twofold. First, this work highlights the different nature of edge-based covering problems, in comparison with node-based covering problems. Informally, in a regular port-numbered graph, all

\begin{tabular}{|c|c|c|c|}
\hline Graph family & Approx. ratio & $\begin{array}{l}\text { Lower bound } \\
\text { Upper bound }\end{array}$ & Time \\
\hline \multicolumn{4}{|c|}{ d-regular graphs: } \\
\hline$d=1,3, \ldots$ & $4-\frac{6}{d+1}$ & $\begin{array}{l}\text { Theorem } 2 \\
\text { Theorem } 4\end{array}$ & $O\left(d^{2}\right)$ \\
\hline$d=2,4, \ldots$ & $4-\frac{2}{d}$ & $\begin{array}{l}\text { Theorem } 1 \\
\text { Theorem } 3\end{array}$ & $O(1)$ \\
\hline \multicolumn{4}{|c|}{ graphs with maximum degree $\Delta:$} \\
\hline$\Delta=1$ & 1 & $\begin{array}{l}\text { trivial } \\
\text { trivial }\end{array}$ & $O(1)$ \\
\hline$\Delta=3,5, \ldots$ & $4-\frac{2}{\Delta-1}$ & $\begin{array}{l}\text { Corollary } 1 \\
\text { Theorem } 5\end{array}$ & $O\left(\Delta^{2}\right)$ \\
\hline$\Delta=2,4,$. & $4-\frac{2}{\Delta}$ & $\begin{array}{l}\text { Corollary } 1 \\
\text { Theorem } 5\end{array}$ & $O\left(\Delta^{2}\right)$ \\
\hline
\end{tabular}

Table 1: Approximability of edge dominating sets: the best possible approximation ratios that can be achieved by any deterministic distributed algorithm in the port-numbering model.

nodes may look identical from the perspective of a distributed algorithm, but all edges do not look identical to each other. Tight lower bound constructions for covering problems such as vertex covers and dominating sets are typically trivial: a cycle or a complete graph will do. This is not the case with edge-based problems.

Second, this work gives yet another example of the close connection between the port-numbering model and local algorithms. In a strictly local algorithm, the running time does not depend on the number of nodes [18, 22]. Even though the negative results hold for any algorithm, regardless of its running time, the matching positive results are local algorithms: the running times depend on the parameters $d$ and $\Delta$, but they are independent of $n$. Indeed, these algorithms are the best known deterministic local algorithms for the edge dominating set problem - it is not known if a better approximation ratio can be achieved in constant time with the help of unique node identifiers.

\section{PRELIMINARIES}

Let $\mathcal{G}$ be a simple, undirected graph with the node set $V_{\mathcal{G}}$ and the edge set $E_{\mathcal{G}}$. An edge $e=\{u, v\} \in E_{\mathcal{G}}$ is said to cover the nodes $u$ and $v$, and an edge $e_{1} \in E_{\mathcal{G}}$ is said to dominate any edge $e_{2} \in E_{\mathcal{G}}$ that is adjacent to $e_{1}$, including $e_{1}$ itself. These terms are generalised to sets of edges and nodes in a natural manner: for example, a set $C \subseteq E_{\mathcal{G}}$ of edges covers a set of nodes $X \subseteq V_{\mathcal{G}}$ if for each $v \in X$ there is an $e \in C$ that covers $v$. An edge cover is a set $C \subseteq E_{\mathcal{G}}$ that covers $V_{\mathcal{G}}$ and an edge dominating set is a set $D \subseteq E_{\mathcal{G}}$ that dominates $E_{\mathcal{G}}$.

A set $M \subseteq E_{\mathcal{G}}$ is a matching if each node $v \in V_{\mathcal{G}}$ is incident to at most one edge of $M$. More generally, a set $M \subseteq E_{\mathcal{G}}$ is a $k$-matching if each node $v \in V_{\mathcal{G}}$ is incident to at most $k$ edges in $M$; put otherwise, a subgraph induced by a $k$-matching is a graph of maximum degree at most $k$. In particular, the subgraph induced by a 2-matching consists of paths and cycles. A matching is maximal if it is not a proper subgraph of a matching. 

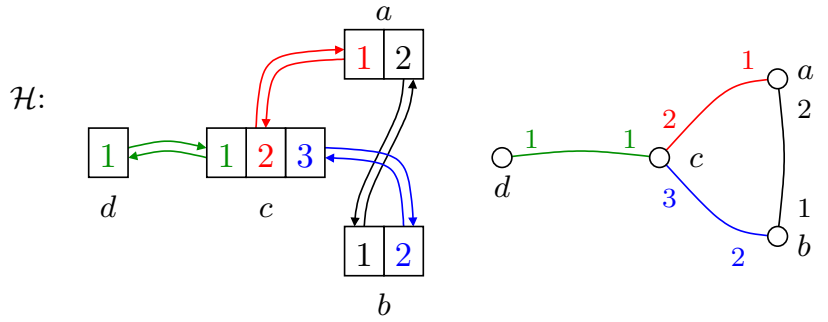

$\mathcal{M}$

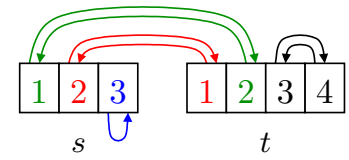

(a)

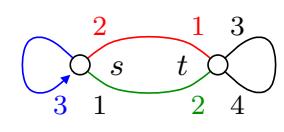

(b)

Figure 2: Examples of port-numbered graphs: a simple graph $\mathcal{H}$ and a multigraph $\mathcal{M}$. For example, $V_{\mathcal{M}}=\{s, t\}, d_{\mathcal{M}}(s)=3, d_{\mathcal{M}}(t)=4$, and the involution $p_{\mathcal{M}} \operatorname{maps}(s, 1) \leftrightarrow(t, 2),(s, 2) \leftrightarrow(t, 1),(s, 3) \mapsto(s, 3)$, and $(t, 3) \leftrightarrow(t, 4)$. (a) Ports (boxes) and connections (arrows). (b) Nodes (circles), undirected edges (lines), and directed edges (arrows).

A $k$-factor of $\mathcal{G}$ is a $k$-regular spanning subgraph $\mathcal{H}$ of $\mathcal{G}$ : we have the same node set $V_{\mathcal{H}}=V_{\mathcal{G}}$, and each node $v \in V_{\mathcal{H}}$ has degree $k$ in $\mathcal{H}$. For example, a 1 -factor forms a perfect matching, and a 2 -factor is a collection of disjoint cycles that $\operatorname{span} V_{\mathcal{G}}$.

A $k$-factorisation of $\mathcal{G}$ is a collection $\mathcal{G}(1), \mathcal{G}(2), \ldots, \mathcal{G}(c)$ of $k$-factors of $\mathcal{G}$ such that each edge $e \in E_{\mathcal{G}}$ is in exactly one $E_{\mathcal{G}(i)}$; that is, a $k$-factorisation partitions the edge set into $k$-factors. For example, a 1 -factorisation of a $d$-regular graph $\mathcal{G}$ can be interpreted as a $d$-colouring of the edges of $\mathcal{G}$ : each factor is a colour class.

Not all graphs admit a $k$-factorisation; an obvious necessary condition is that the graph $\mathcal{G}$ has to be $c k$-regular for some $c$. In the case of 1-factorisations, this condition is not sufficient: there are regular graphs that cannot be 1-factorised (e.g., an odd cycle). However, in the case of 2 -factorisations, this condition turns out to be sufficient. A 120-year-old result due to Petersen [20] shows that any $2 k$ regular graph admits a 2 -factorisation - see, e.g., Diestel [11, p. 39] for a modern proof.

\subsection{Port-Numbered Graphs}

A port-numbered graph $\mathcal{G}$ is defined by a set of nodes $V_{\mathcal{G}}$ and two functions, $d_{\mathcal{G}}: V_{\mathcal{G}} \rightarrow \mathbb{N}$ and $p_{\mathcal{G}}: P_{\mathcal{G}} \rightarrow P_{\mathcal{G}}$, where

$$
\begin{aligned}
& P_{\mathcal{G}}=\left\{(v, i): v \in V_{\mathcal{G}}, i \in P_{\mathcal{G}}(v)\right\}, \\
& P_{\mathcal{G}}(v)=\left\{1,2, \ldots, d_{\mathcal{G}}(v)\right\} .
\end{aligned}
$$

It is required that $p_{\mathcal{G}}$ is an involution, i.e., a bijection that is its own inverse.

The integer $d_{\mathcal{G}}(v)$ is called the degree of the node $v \in V_{\mathcal{G}}$. Each $(v, i) \in P_{\mathcal{G}}$ is a port. If $p_{\mathcal{G}}(v, i)=(u, j)$, we say that the port $i$ of $v$ is connected to the port $j$ of $u$. Figure 2a shows two examples of port-numbered graphs.

Given the involution $p_{\mathcal{G}}$, we can define the multiset of edges $E_{\mathcal{G}}$ as follows: For each pair of ports $(v, i),(u, j) \in$ $P_{\mathcal{G}}$ with $p_{\mathcal{G}}(v, i)=(u, j)$ and $(v, i) \neq(u, j)$, we have an undirected edge $\{v, u\} \in E_{\mathcal{G}}$, and for each fixed point $(v, i) \in$
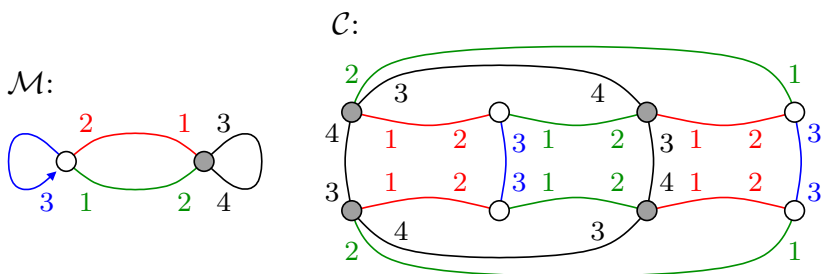

Figure 3: An example of a covering graph. The simple port-numbered graph $\mathcal{C}$ is a covering graph of the multigraph $\mathcal{M}$. The covering map $f$ maps each grey node of $\mathcal{C}$ to the grey node of $\mathcal{M}$, and each white node of $\mathcal{C}$ to the white node of $\mathcal{M}$.

$P_{\mathcal{G}}$ with $p_{\mathcal{G}}(v, i)=(v, i)$, we have a directed loop $(v, v) \in E_{\mathcal{G}}$; see Figure $2 \mathrm{~b}$ for an illustration.

This way we can interpret any port-numbered graph $\mathcal{G}$ as a graph with the node set $V_{\mathcal{G}}$ and the edge set $E_{\mathcal{G}}$, and we can also apply the usual graph-theoretic terminology; for example, a port-numbered graph $\mathcal{G}$ is simple if the edge set $E_{\mathcal{G}}$ does not contain loops or multiple parallel edges. Conversely, we can take any undirected graph $\mathcal{G}$ with the node set $V_{\mathcal{G}}$ and the edge set $E_{\mathcal{G}}$, and turn $\mathcal{G}$ into a portnumbered graph by constructing an involution $p_{\mathcal{G}}$ that is compatible with $E_{\mathcal{G}}$.

\subsection{Model of Computation}

In a synchronous distributed algorithm, computation proceeds in synchronous communication rounds. In each round, the following operations are performed in a port-numbered graph $\mathcal{G}$ : (i) each node performs local computation, (ii) each node $v \in V_{\mathcal{G}}$ sends one message to each port $i \in P_{\mathcal{G}}(v)$, and (iii) each node $v \in V$ receives one message from each port $i \in P_{\mathcal{G}}(v)$. The involution $p_{\mathcal{G}}$ indicates how the messages are routed: if $p_{\mathcal{G}}(v, i)=(u, j)$, then the message sent by $v$ to its port $i$ is received by $u$ from its port $j$.

All nodes run the same deterministic distributed algorithm $\mathcal{A}$. Initially, each node $v \in V_{\mathcal{G}}$ knows only its degree $d_{\mathcal{G}}(v)$. After each round, a node may decide to stop computation and announce its output. The running time of the algorithm $\mathcal{A}$ is the maximum number of synchronous rounds until all nodes have stopped.

When we use a distributed algorithm $\mathcal{A}$ to find an edge dominating set $D$ in a simple port-numbered graph $\mathcal{G}$, we assume that each node $v \in V_{\mathcal{G}}$ outputs a subset $X(v) \subseteq$ $P_{\mathcal{G}}(v)$ of port numbers; if $i \in X(v)$ and $p_{\mathcal{G}}(v, i)=(u, j)$ then the edge $\{u, v\}$ is in the set $D$. Naturally, we require that the output is internally consistent: if $i \in X(v)$ and $p_{\mathcal{G}}(v, i)=(u, j)$, then we must also have $j \in X(u)$.

\subsection{Covering Maps}

Let $\mathcal{G}$ and $\mathcal{H}$ be two port-numbered graphs. A surjection $f: V_{\mathcal{H}} \rightarrow V_{\mathcal{G}}$ is a covering map from $\mathcal{H}$ to $\mathcal{G}$ if (i) it preserves the degrees, i.e., $d_{\mathcal{H}}(v)=d_{\mathcal{G}}(f(v))$ for all $v \in V_{\mathcal{H}}$, and (ii) it preserves the connections, i.e., $p_{\mathcal{H}}(v, i)=(u, j)$ implies $p_{\mathcal{G}}(f(v), i)=(f(u), j)$ for all $(v, i) \in P_{\mathcal{H}}$. If there exists a covering map from $\mathcal{H}$ to $\mathcal{G}$, then $\mathcal{H}$ is a covering graph of $\mathcal{G}$. See Figure 3 for an example.

A key observation is that if we apply any deterministic distributed algorithm $\mathcal{A}$ both in the port-numbered graph $\mathcal{G}$ and in its covering graph $\mathcal{H}$, then the output of a node $v \in V_{\mathcal{H}}$ is necessarily identical to the output of the node 

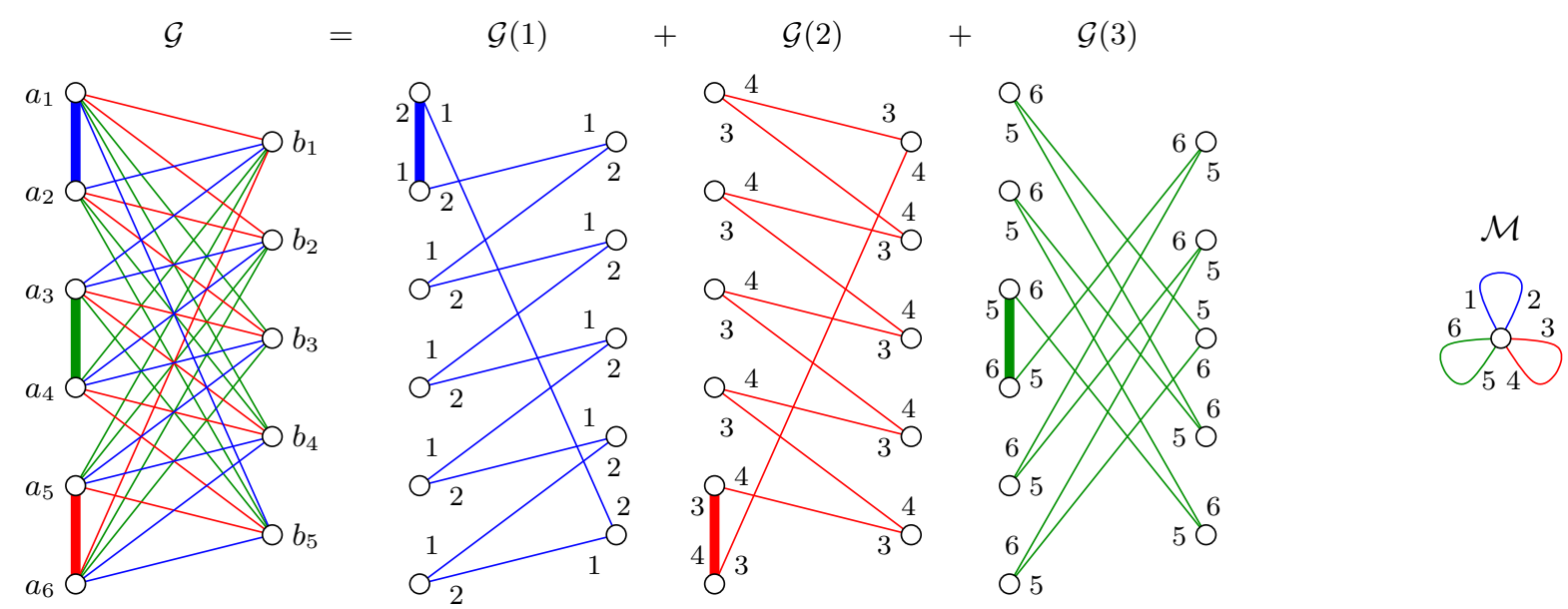

Figure 4: The graph $\mathcal{G}$ constructed in the proof of Theorem 1 for $d=6$. The thick lines indicate the optimal edge dominating set $S$. The graph $\mathcal{G}$ is a covering graph of $\mathcal{M}$.

$f(v) \in V_{\mathcal{G}}[2,7,22,24]$. To see this, note that the initial state of a node $v \in V_{\mathcal{H}}$ is identical to the initial state of the node $f(v) \in V_{\mathcal{G}}$, as both of them run the same algorithm $\mathcal{A}$. Now assume inductively that before the communication round $t$, for each $v \in V_{\mathcal{H}}$ the local state of $v$ in $\mathcal{H}$ is the same as the local state of $f(v)$ in $\mathcal{G}$. Then during the round $t$, for each $(v, i) \in P_{\mathcal{H}}$ the message sent to the port $(v, i)$ in $\mathcal{H}$ equals the message sent to $(f(v), i)$ in $\mathcal{G}$. Since the covering map preserves the connections, it follows that for each $(v, i) \in$ $P_{\mathcal{H}}$ the message received from the port $(v, i)$ in $\mathcal{H}$ equals the message received from $(f(v), i)$ in $\mathcal{G}$; hence after the round $t$, the local state of $v \in V_{\mathcal{H}}$ is identical to the local state of $f(v) \in V_{\mathcal{G}}$. Whenever the node $v$ decides to stop and announce its output, the node $f(v)$ also stops and produces the same output.

\section{LOWER BOUND CONSTRUCTION: EVEN DEGREE}

In this section we prove the following theorem.

THEOREM 1. For each $d=2,4, \ldots$, there is a d-regular port-numbered graph $\mathcal{G}$ such that no deterministic distributed algorithm can achieve a better approximation ratio than $4-2 / d$ for the minimum edge dominating set problem in $\mathcal{G}$.

\subsection{Graph}

The graph $\mathcal{G}$ is constructed as follows (see Figure 4 for an illustration in the case $d=6$ ). The node set is $V_{\mathcal{G}}=A \cup B$ where

$$
A=\left\{a_{1}, a_{2}, \ldots, a_{d}\right\}, \quad B=\left\{b_{1}, b_{2}, \ldots, b_{d-1}\right\} .
$$

The edge set is $E_{\mathcal{G}}=S \cup T$ where

$$
\begin{aligned}
& S=\left\{\left\{a_{1}, a_{2}\right\},\left\{a_{3}, a_{4}\right\}, \ldots,\left\{a_{d-1}, a_{d}\right\}\right\}, \\
& T=\left\{\left\{a_{i}, b_{j}\right\}: a_{i} \in A, b_{j} \in B\right\} .
\end{aligned}
$$

The graph $\mathcal{G}$ is $d$-regular. The subgraph induced by $S$ is a matching and the subgraph induced by $T$ is the complete bipartite graph $K_{d, d-1}$. By construction, $S$ is an edge dominating set: each edge in $T$ is adjacent to an edge in $S$. Moreover, $S$ is an optimal edge dominating set, since
$\left|E_{\mathcal{G}}\right|=(2 d-1)|S|$ and each edge can dominate at most $2 d-1$ edges.

\subsection{Port Numbering}

Let $k=d / 2$. Since $\mathcal{G}$ is $2 k$-regular, we can 2 -factorise it; let the factors be $\mathcal{G}(1), \mathcal{G}(2), \ldots, \mathcal{G}(k)$ - see Figure 4 for an example. Each subgraph $\mathcal{G}(i)$ consists of cycles. Let $\mathcal{H}(i)$ be an orientation of $\mathcal{G}(i)$ that consists of directed cycles that is, for each $\{u, v\} \in E_{\mathcal{G}(i)}$ there is either $(u, v)$ or $(v, u)$ in $E_{\mathcal{H}(i)}$, and the outdegree and indegree of each node $v$ of $\mathcal{H}(i)$ is 1 .

Now we are ready to define a port numbering $p_{\mathcal{G}}$ for $\mathcal{G}$. For each $i=1,2, \ldots, k$ and for each $(u, v) \in E_{\mathcal{H}(i)}$, we set $p_{\mathcal{G}}(u, 2 i-1)=(v, 2 i)$ and conversely $p_{\mathcal{G}}(v, 2 i)=(u, 2 i-1)$; see Figure 4.

\subsection{Covering Map}

Let $\mathcal{M}$ be a port-numbered multigraph with one node $V_{\mathcal{M}}=\{x\}$ of degree $d_{\mathcal{M}}(x)=2 k$. The involution $p_{\mathcal{M}}$ maps $(x, 2 i-1) \leftrightarrow(x, 2 i)$ for each $i=1,2, \ldots, k$. See Figure 4 for an illustration in the case $d=6$. Define the function $f: V_{\mathcal{G}} \rightarrow V_{\mathcal{M}}$ by setting $f(v)=x$ for all $v \in V_{\mathcal{G}}$; it can be checked that $f$ is a covering map from $\mathcal{G}$ to $\mathcal{M}$.

\subsection{Approximation Ratio}

Let $\mathcal{A}$ be a deterministic distributed algorithm that finds an edge dominating set in any port-numbered $2 k$-regular graph, and apply $\mathcal{A}$ to $\mathcal{G}$; let $D$ be the edge dominating set produced by $\mathcal{A}$. Since $D \neq \emptyset$, there is an edge $e \in D$; let $\mathcal{G}(i)$ be the 2 -factor with $e \in E_{\mathcal{G}(i)}$. Hence there is a node $a$ that outputs a set $X(a)$ which contains the port number $2 i-1$ and another node $b$ that outputs a set $X(b)$ which contains the port number $2 i$.

The covering map $f$ shows that all nodes of $\mathcal{G}$ produce the same output. Hence all nodes $v \in V_{\mathcal{G}}$ output a set $X(v)$ with $\{2 i-1,2 i\} \subseteq X(v)$, and the dominating set $D$ has to contain all edges of the factor $\mathcal{G}(i)$. We conclude that the approximation ratio of $\mathcal{A}$ is at least

$$
\frac{|D|}{|S|} \geq \frac{\left|E_{\mathcal{G}(i)}\right|}{|S|}=\frac{\left|V_{\mathcal{G}}\right|}{|S|}=\frac{4 k-1}{k},
$$

which completes the proof of Theorem 1. 

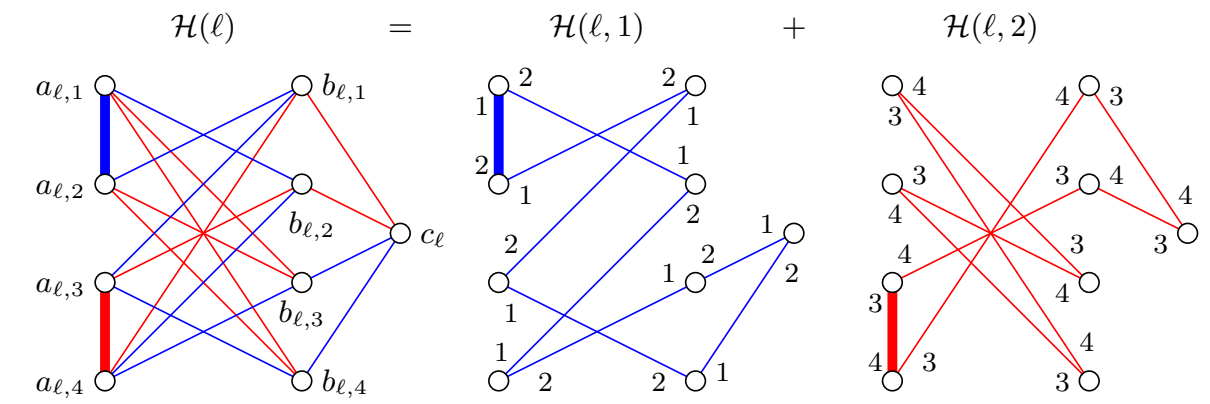

Figure 5: The component $\mathcal{H}(\ell)$ used in the proof of Theorem 2 for $d=5$. The thick lines indicate the set $S(\ell)$.

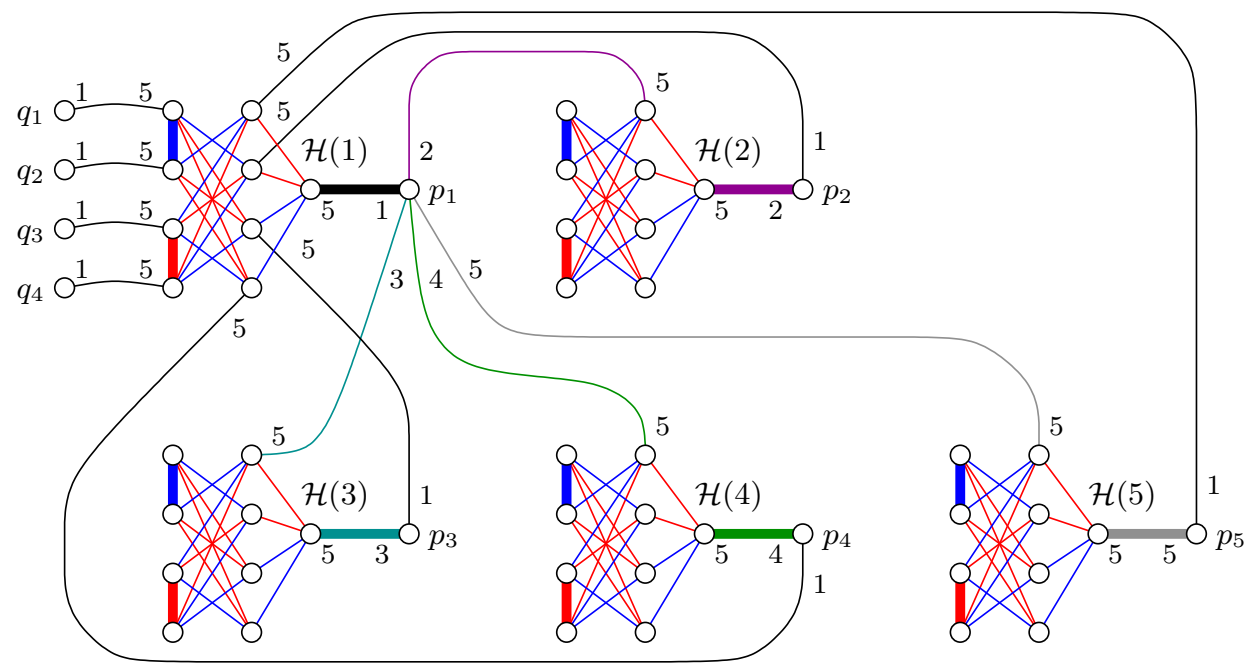

Figure 6: The graph $\mathcal{G}$ constructed in the proof of Theorem 2 for $d=5$. The thick lines indicate the optimal dominating set $D^{*}$. For clarity, only a subset of edge is shown: edges of each $\mathcal{H}(\ell)$, edges connected to $\mathcal{H}(1)$, edges connected to $p_{1}$, and edges in $Y$. The components $\mathcal{H}(\ell)$ are illustrated in Figure 5 in more detail.

\section{LOWER BOUND CONSTRUCTION: ODD DEGREE}

In this section we prove the following theorem.

TheOREM 2. For each $d=1,3, \ldots$, there is a d-regular port-numbered graph $\mathcal{G}$ such that no deterministic distributed algorithm can achieve a better approximation ratio than $4-6 /(d+1)$ for minimum edge dominating sets in $\mathcal{G}$.

\subsection{Graph}

Let $k=(d-1) / 2$. For each $\ell=1,2, \ldots, d$, we first construct a $2 k$-regular graph $\mathcal{H}(\ell)$ as follows (see Figure 5 for an illustration in the case $d=5)$. The node set is $V_{\mathcal{H}(\ell)}=A(\ell) \cup B(\ell) \cup C(\ell)$ where

$$
\begin{aligned}
& A(\ell)=\left\{a_{\ell, 1}, a_{\ell, 2}, \ldots, a_{\ell, 2 k}\right\}, \quad C(\ell)=\left\{c_{\ell}\right\}, \\
& B(\ell)=\left\{b_{\ell, 1}, b_{\ell, 2}, \ldots, b_{\ell, 2 k}\right\} .
\end{aligned}
$$

The edge set is $E_{\mathcal{H}(\ell)}=R(\ell) \cup S(\ell) \cup T(\ell)$ where

$$
\begin{aligned}
R(\ell) & =\left\{\left\{c_{\ell}, b_{\ell, i}\right\}: b_{\ell, i} \in B(\ell)\right\}, \\
S(\ell) & =\left\{\left\{a_{\ell, 1}, a_{\ell, 2}\right\},\left\{a_{\ell, 3}, a_{\ell, 4}\right\}, \ldots,\left\{a_{\ell, 2 k-1}, a_{\ell, 2 k}\right\}\right\}, \\
T(\ell) & =\left\{\left\{a_{\ell, i}, b_{\ell, j}\right\}: a_{\ell, i} \in A(\ell), b_{\ell, j} \in B(\ell), i \neq j\right\} .
\end{aligned}
$$

The subgraph induced by $R(\ell)$ is a star, the subgraph induced by $S(\ell)$ is a matching, and the subgraph induced by
$T(\ell)$ is a crown graph (a complete bipartite graph minus a perfect matching).

Since the graph $\mathcal{H}(\ell)$ is $2 k$-regular, we can again find a 2 -factorisation and hence construct a port numbering $p_{\mathcal{H}(\ell)}$ so that for each node $u$ and each $i=1,2, \ldots, 2 k$, the port $2 i-1$ of $u$ is connected to the port $2 i$ of an adjacent node $v$ and vice versa; see Figure 5 for an example.

The port-numbered graph $\mathcal{G}$ contains the port-numbered components $\mathcal{H}(\ell)$ for each $\ell=1,2, \ldots, d$ as subgraphs. The node set of $\mathcal{G}$ consists of the node sets of the components $\mathcal{H}(\ell)$ and the sets

$$
P=\left\{p_{1}, p_{2}, \ldots, p_{d}\right\}, \quad Q=\left\{q_{1}, q_{2}, \ldots, q_{2 k}\right\} .
$$

We create the following connections, in addition to those inherited from the components $\mathcal{H}(\ell)$ :

$$
\begin{aligned}
\left(p_{\ell}, \ell\right) & \leftrightarrow\left(c_{\ell}, d\right) \quad \forall \ell=1,2, \ldots, d, \\
\left(p_{i}, \ell\right) & \leftrightarrow\left(b_{\ell, i}, d\right) \quad \forall \ell=1,2, \ldots, d, i=1,2, \ldots, 2 k, i \neq \ell, \\
\left(p_{d}, \ell\right) & \leftrightarrow\left(b_{\ell, \ell}, d\right) \quad \forall \ell=1,2, \ldots, d, \\
\left(q_{i}, \ell\right) & \leftrightarrow\left(a_{\ell, i}, d\right) \quad \forall \ell=1,2, \ldots, d, i=1,2, \ldots, 2 k .
\end{aligned}
$$

See Figure 6 for an illustration in the case $d=5$. Note, in particular, that each edge that joins a node $u \in P \cup Q$ to a node $v \in V_{\mathcal{H}(\ell)}$ connects the port $\ell$ of $u$ to the port $d$ of $v$. 


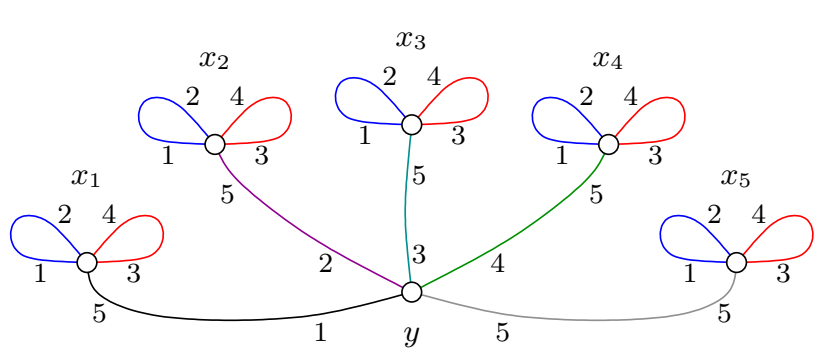

Figure 7: The multigraph $\mathcal{M}$ from the proof of Theorem 2. The graph $\mathcal{G}$ in Figure 6 is a covering graph of $\mathcal{M}$.

\subsection{Optimal Solution}

Define the edge sets

$$
Y=\left\{\left\{p_{\ell}, c_{\ell}\right\}: \ell=1,2, \ldots, d\right\}, \quad D^{*}=Y \cup \bigcup_{\ell} S(\ell) .
$$

Now $D^{*}$ is an optimal edge dominating set for the graph $\mathcal{G}$; each edge $e \notin D^{*}$ is adjacent to exactly one edge in $D^{*}$. By construction, $\left|D^{*}\right|=(k+1) d$.

\subsection{Covering Map}

Let $\mathcal{M}$ be a port-numbered multigraph with the node set

$$
V_{\mathcal{M}}=\left\{x_{1}, x_{2}, \ldots, x_{d}, y\right\} .
$$

All nodes $v \in V_{\mathcal{M}}$ have degree $d_{\mathcal{M}}(v)=d$. The involution $p_{\mathcal{M}}$ maps

$$
\begin{array}{rlrl}
\left(x_{\ell}, 2 i-1\right) & \leftrightarrow\left(x_{\ell}, 2 i\right) & \forall \ell=1,2, \ldots, d, i=1,2, \ldots, k \\
(y, \ell) & \leftrightarrow\left(x_{\ell}, d\right) \quad \forall \ell=1,2, \ldots, d .
\end{array}
$$

See Figure 7 for an illustration in the case $d=5$.

Define the function $f: V_{\mathcal{G}} \rightarrow V_{\mathcal{M}}$ as follows:

$$
\begin{array}{ll}
f(v)=x_{\ell} & \text { for each } \ell=1,2, \ldots, d, v \in V_{\mathcal{H}(\ell)}, \\
f(v)=y & \text { for each } v \in P \cup Q .
\end{array}
$$

It can be checked that $f$ is a covering map from $\mathcal{G}$ to $\mathcal{M}$. Hence we have partitioned the node set of $\mathcal{G}$ in $d+1$ equivalence classes and the edge set of $\mathcal{G}$ in $(k+1) d$ equivalence classes.

\subsection{Approximation Ratio}

Assume that $\mathcal{A}$ is a deterministic distributed algorithm that finds an edge dominating set in any port-numbered $d$ regular graph. Apply $\mathcal{A}$ to $\mathcal{G}$; let $D$ be the edge dominating set produced by $\mathcal{A}$. Consider an $\ell \in\{1,2, \ldots, d\}$. To dominate $S(\ell)$, there must exist a node $a_{\ell, i} \in A(\ell)$ that is incident to an edge $e \in D$; in particular, the output $X\left(a_{\ell, i}\right)$ is nonempty. Since $f\left(a_{\ell, i}\right)=x_{\ell}$ and $f^{-1}\left(x_{\ell}\right)=V_{\mathcal{H}(\ell)}$, we conclude that all nodes $v \in V_{\mathcal{H}(\ell)}$ produce the same non-empty set of port numbers, let us call it $X_{\ell}$.

If $d \in X_{\ell}$, then $D$ contains $2 d-1$ edges that join $P \cup Q$ to $\mathcal{H}(\ell)$. Otherwise $D$ contains all edges in one of the 2 -factors of $\mathcal{H}(\ell)$; for example, if $1 \in X_{\ell}$, then also $2 \in X_{\ell}$ and $D$ contains all edges of $\mathcal{H}(\ell)$ that connect the port 1 of a node to the port 2 of another node; since $\mathcal{H}(\ell)$ has $2 d-1$ nodes, the 2-factor contains $2 d-1$ edges.

This way we can find $d$ disjoint sets of edges that are contained in $D$, one for each $\ell$, and each set consists of $2 d-1$ edges; hence $|D| \geq(2 d-1) d$. We conclude that the approximation ratio of $\mathcal{A}$ is at least

$$
\frac{|D|}{\left|D^{*}\right|} \geq \frac{(2 d-1) d}{(k+1) d}=\frac{4 k+1}{k+1},
$$

which completes the proof of Theorem 2 .

\section{DISTINGUISHABLE NEIGHBOURS}

This section introduces concepts and lemmas that are used in Sections 6 and 7 to facilitate algorithm design. Throughout this section, let $\mathcal{G}$ be a simple port-numbered graph. Then for each edge $\{v, u\} \in E_{\mathcal{G}}$ there are unique port numbers $i$ and $j$ such that $p_{\mathcal{G}}(v, i)=(u, j)$; we use the notation $\ell_{\mathcal{G}}(v, u)=i$ and $\ell_{\mathcal{G}}(u, v)=j$ to refer to these port numbers.

The label pair of an edge $\{v, u\} \in E_{\mathcal{G}}$ is the unordered pair $\ell_{\mathcal{G}}\{u, v\}=\left\{\ell_{\mathcal{G}}(v, u), \ell_{\mathcal{G}}(u, v)\right\}$. The set of uniquely labelled edges of $v \in V_{\mathcal{G}}$ consists of the edges incident to $v$ whose label pair is different from the label pair of any other edge incident to $v$. We say that the node $u$ is the distinguishable neighbour of $v \in V_{\mathcal{G}}$ if $\{v, u\} \in E_{\mathcal{G}}$ is the uniquely labelled edge of $v$ that minimises the port number $\ell_{\mathcal{G}}(v, u)$.

Whenever a node has at least one uniquely labelled edge, then it also has exactly one distinguishable neighbour. For example, in the graph $\mathcal{H}$ of Figure 2, $a$ is the distinguishable neighbour of $b$, and $d$ is the distinguishable neighbour of $c$. However, the node $a$ does not have any uniquely labelled edges, and hence it does not have a distinguishable neighbour, either. A key observation is that this can happen only if the node has an even degree (see Figure 8a for an example of a 3-regular graph: all nodes have distinguishable neighbours).

Lemma 1. Let $v \in V_{\mathcal{G}}$ be a node with an odd degree. Then the node $v$ has a distinguishable neighbour.

Proof. For all $i$ and $j$, there are at most two edges incident to $v$ with the label pair $\{i, j\}$ : one connected to the port $i$ and the other connected to the port $j$. Discard such pairs of edges with duplicate label pairs; since $d_{\mathcal{G}}(v)$ is odd, at least one edge with a unique label pair is retained.

Let $M_{\mathcal{G}}(i, j)$ consist of all edges $\{v, u\} \in \mathcal{G}$ such that $p_{\mathcal{G}}(v, i)=(u, j)$ and $u$ is the distinguishable neighbour of $v$; see Figure $8 \mathrm{~b}$ for an illustration.

Lemma 2. For all $i$ and $j, M_{\mathcal{G}}(i, j)$ is a matching in $\mathcal{G}$.

Proof. To reach a contradiction, assume that $\{v, t\}$ and $\{v, u\}$ are two distinct but adjacent edges in $M_{\mathcal{G}}(i, j)$ for some $i, j$. We must have $\ell_{\mathcal{G}}(v, t) \neq \ell_{\mathcal{G}}(v, u)$ and $\ell_{\mathcal{G}}(v, t)$, $\ell_{\mathcal{G}}(v, u) \in\{i, j\}$; in particular, $i \neq j$. W.l.o.g., let $\ell_{\mathcal{G}}(v, t)=$ $i$ and $\ell_{\mathcal{G}}(v, u)=j$. From the definition of $M_{\mathcal{G}}(i, j)$, it follows that $t$ has to be the distinguishable neighbour of $v$, and $v$ has to be the distinguishable neighbour of $u$; moreover, $\ell_{\mathcal{G}}\{v, t\}=\ell_{\mathcal{G}}\{v, u\}=\{i, j\}$. However, then $\{v, t\}$ cannot be a uniquely labelled edge of $v$, and $t$ cannot be the distinguishable neighbour of $v$.

Note that the sets $M_{\mathcal{G}}(i, j)$ can be constructed by a distributed algorithm in constant time. To rephrase Lemmas 1 and 2, we can construct a collection of matchings whose union covers all nodes with an odd degree. Note that the matchings $M_{\mathcal{G}}(i, j)$ are not necessarily disjoint; we may have $i \neq j$ and $M_{\mathcal{G}}(i, j) \cap M_{\mathcal{G}}(j, i) \neq \emptyset$. 


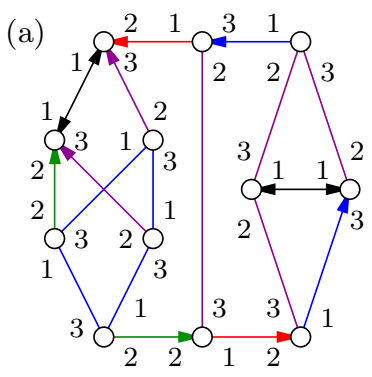

(b)
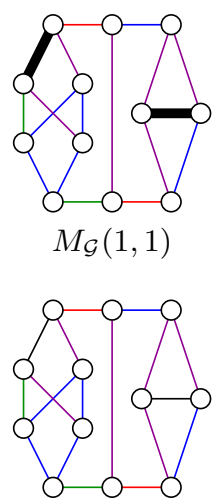

$M_{\mathcal{G}}(2,1)$

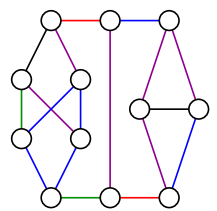

$M_{\mathcal{G}}(3,1)$
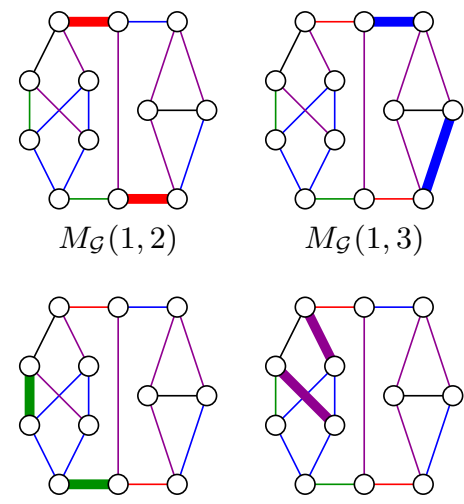

$M_{\mathcal{G}}(2,3)$

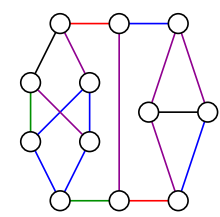

$M_{\mathcal{G}}(3,2)$

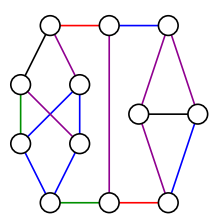

$M_{\mathcal{G}}(3,3)$ (c)

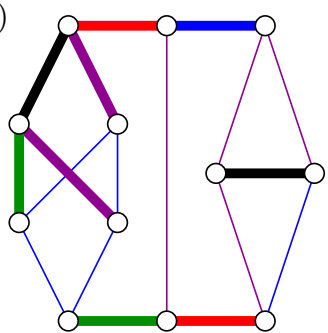

(d)

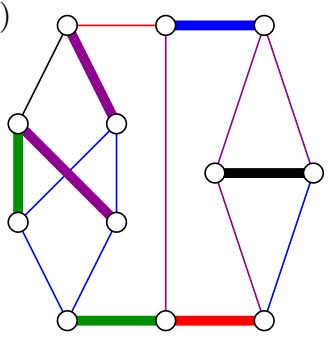

Figure 8: (a) A 3-regular port-numbered graph $\mathcal{G}$; an arrow from $u$ to $v$ indicates that $v$ is the distinguishable neighbour of $u$. (b) The matchings $M_{\mathcal{G}}(i, j)$. (c) Phase I of the algorithm from Theorem 4. (d) Phase II.

\section{OPTIMAL ALGORITHMS FOR REGULAR GRAPHS}

Let us first present a trivial algorithm that shows that the lower bound of Theorem 1 is tight.

THEOREM 3. There is a deterministic distributed $O(1)$ time algorithm that finds a factor $4-2 / d$ approximation of a minimum edge dominating set in any $d$-regular portnumbered graph for any $d=1,2, \ldots$.

Proof. The algorithm outputs all edges that are connected to a port with port number 1 .

Let $D$ be the output of the algorithm in a port-numbered graph $\mathcal{G}$. First observe that $D$ is a feasible solution, as it covers all nodes and hence dominates all edges. To analyse the approximation ratio, note that the number of edges in the solution $D$ is at most $\left|V_{\mathcal{G}}\right|$. Since the graph is $d$-regular, we have $d\left|V_{\mathcal{G}}\right|=2\left|E_{\mathcal{G}}\right|$. Each edge in an optimal solution $D^{*}$ dominates at most $2 d-1$ edges, i.e., $\left|E_{\mathcal{G}}\right| \leq(2 d-1)\left|D^{*}\right|$. Thus the approximation factor is $|D| /\left|D^{*}\right| \leq 4-2 / d$.

The following result shows that the lower bound of Theorem 2 is tight as well.

TheOREM 4. There is a deterministic distributed $O\left(d^{2}\right)$ time algorithm that finds a factor $4-6 /(d+1)$ approximation of a minimum edge dominating set in any d-regular portnumbered graph for any $d=1,3, \ldots$.

Proof. Let $\mathcal{G}$ be a $d$-regular port-numbered graph. The algorithm constructs an edge dominating set $D \subseteq E_{\mathcal{G}}$ in two phases, both of which can be implemented in $O\left(d^{2}\right)$ communication rounds. Initially, set $D \leftarrow \emptyset$.

In phase $\mathrm{I}$, we consider each pair $(i, j)$ with $i, j \in\{1,2$, $\ldots, d\}$ sequentially (in an arbitrary order), and for each pair $(i, j)$ we process all distinguishable edges $e \in M_{\mathcal{G}}(i, j)$ in parallel: if both endpoints of $e$ are already covered by $D$, we ignore $e$, otherwise we add $e$ to $D$. See Figure $8 \mathrm{c}$ for an example.

In phase II, we consider again each pair $(i, j)$ sequentially, and for each pair $(i, j)$ we process all edges $e \in D \cap M_{\mathcal{G}}(i, j)$ in parallel: if both endpoints of $e$ are covered by $D \backslash\{e\}$, remove $e$ from $D$. Finally, the algorithm outputs the set $D$; see Figure $8 \mathrm{~d}$ for an example.

Recall that each set $M_{\mathcal{G}}(i, j)$ is a matching; hence the decisions related to the edges in $M_{\mathcal{G}}(i, j)$ are independent of each other and can be performed in parallel. Phase I constructs a spanning forest $D$ : The set $D$ covers the same set of nodes as the union of the sets $M_{\mathcal{G}}(i, j)$, as we ignore only redundant edges; therefore $D$ is an edge cover. Moreover, we never add edges that could close a cycle; hence the subgraph induced by $D$ is a forest.

Phase II removes some redundant edges from $D$; the property that $D$ is an edge cover is preserved throughout phase II. Moreover, phase II guarantees that there cannot be a path of length 3 in the forest $D$ : if there is a path with three edges, both endpoints of the middle edge are covered by other edges. Thus $D$ is a forest of node-disjoint stars. In particular, each tree in $D$ contains at most $d$ edges, and therefore $|D| \leq d\left|V_{\mathcal{G}}\right| /(d+1)$.

It follows that the set $D$ is a feasible solution - an edge cover is an edge dominating set. Moreover,

$$
|D| \leq \frac{d}{d+1}\left|V_{\mathcal{G}}\right|=\frac{2}{d+1}\left|E_{\mathcal{G}}\right| \leq \frac{4 d-2}{d+1}\left|D^{*}\right| .
$$

\section{OPTIMAL ALGORITHMS FOR BOUNDED-DEGREE GRAPHS}

So far we have discussed distributed algorithms for regular graphs; now we turn our attention to bounded-degree graphs. Throughout this section $\Delta$ is a positive integer. 


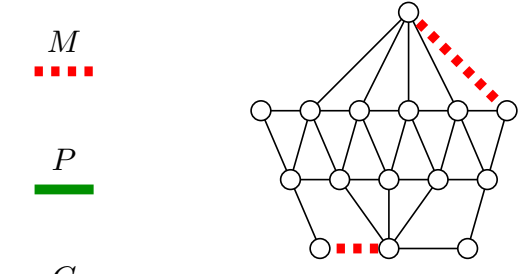

$\underline{C}$
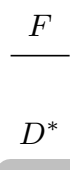

Phase I

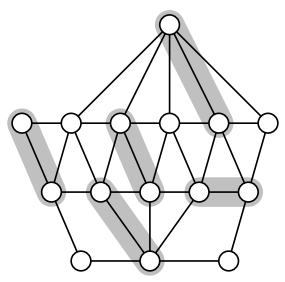

Matching $D^{*}$

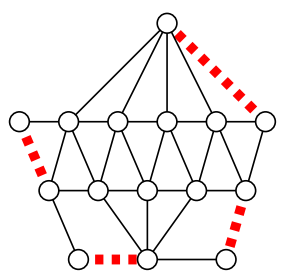

Phase II

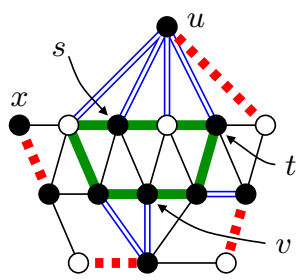

Internal nodes (black) and costs

$c(s)=2$

$c(t)=\frac{3}{2}$

$c(u)=c(v)=1$

$c(x)=\frac{1}{2}$

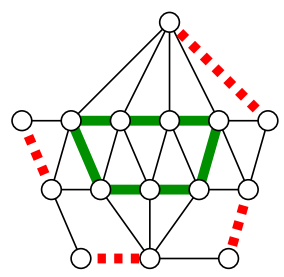

Phase III

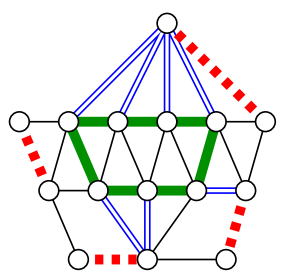

Definition of $C$

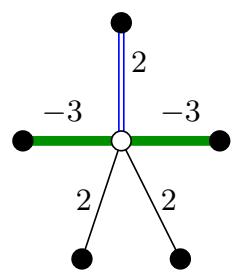

Edge weights

Figure 9: Algorithm from Section 7 (a schematic illustration, not derived from any specific port numbering).

Let $\mathcal{A}$ be a family of algorithms parametrised by $\Delta$, and let $\alpha$ be a real-valued function of $\Delta$. We say that $\mathcal{A}$ finds an $\alpha$-approximation for edge dominating set in bounded-degree graphs if the following holds for every $\Delta$ : if $\mathcal{G}$ is a portnumbered graph such that $d_{\mathcal{G}}(v) \leq \Delta$ for all $v \in V_{\mathcal{G}}$, then the algorithm $\mathcal{A}(\Delta)$ finds an $\alpha(\Delta)$-approximation of a minimum edge dominating set in $\mathcal{G}$.

Obviously $\alpha(\Delta+1) \geq \alpha(\Delta) \geq 1$, and $k$-regular graphs satisfy $d_{\mathcal{G}}(v) \leq k$ by definition. Hence Theorem 1 has the following corollary.

COROLlaRY 1. For any family of algorithms that finds an $\alpha$-approximation for edge dominating set in bounded-degree graphs, we have $\alpha(1) \geq 1$ and $\alpha(2 k+1) \geq \alpha(2 k) \geq 4-1 / k$ for all $k=1,2, \ldots$.

In this section we show that the corollary is tight.

THEOREM 5. There is a family of algorithms that finds an $\alpha$-approximation for edge dominating set in bounded-degree graphs such that $\alpha(1)=1$ and $\alpha(2 k+1)=\alpha(2 k)=4-1 / k$ for all $k=1,2, \ldots$. The running time of $\mathcal{A}(\Delta)$ is $O\left(\Delta^{2}\right)$.

The case $\Delta=1$ is trivial: the optimal edge dominating set consists of all edges. In what follows, let $k \geq 1$ and $\Delta=2 k+1$. We present the algorithm $\mathcal{A}(\Delta)$ and show that $\alpha(\Delta) \leq 4-1 / k$; the claim $\alpha(2 k) \leq 4-1 / k$ then follows by choosing $\mathcal{A}(2 k)=\mathcal{A}(2 k+1)$.

\subsection{Algorithm}

In the algorithm, we will construct two node-disjoint sets of edges: a matching $M$ and a 2-matching $P$. Initially, set $M \leftarrow \emptyset$, and $P \leftarrow \emptyset$. Refer to Figure 9 for an illustration.

In phase $\mathrm{I}$, we consider each pair $(i, j)$ with $i, j \in\{1,2$, $\ldots, \Delta\}$ sequentially, and for each pair $(i, j)$ we process all distinguishable edges $e=\{u, v\} \in M_{\mathcal{G}}(i, j)$ in parallel: if neither $u$ nor $v$ is covered by $M$, we add $e$ to $M$. This phase requires $O\left(\Delta^{2}\right)$ synchronous communication rounds.

In phase II, we consider each $i \in\{2,3, \ldots, \Delta\}$ sequentially. Let $B_{i}$ consist of the edges $\{u, v\} \in E_{\mathcal{G}}$ such that $d_{\mathcal{G}}(u)<$ $d_{\mathcal{G}}(v)=i$ and neither $u$ nor $v$ is covered by $M$. The subgraph of $\mathcal{G}$ induced by $B_{i}$ is bipartite; we can easily 2 -colour it by assigning the black colour to each node $v$ with $d_{\mathcal{G}}(v)=i$ and the white colour to each node $u$ with $d_{\mathcal{G}}(u)<i$. Hence we can also find a maximal matching $M_{i}$ in this subgraph in $O(i)$ rounds [13]:

- Each black node sends proposals to its white neighbours, in the order of increasing port numbers, until a proposal is accepted or the list of white neighbours is exhausted.

- Each white node accepts the first proposal it gets (if any), breaking the ties with its port numbers.

Each edge with an accepted proposal is added to $M_{i}$. After constructing $M_{i}$, set $M \leftarrow M \cup M_{i}$, and proceed with the next value of $i$. In total, phase II requires $O\left(\Delta^{2}\right)$ rounds.

In phase III, we consider the subgraph $\mathcal{H}$ induced by the edges that are not yet covered by $M$. We find a 2 -matching $P$ in $\mathcal{H}$ that dominates all edges in $\mathcal{H}$. This is possible by using a simple $O(\Delta)$-time algorithm [21] that constructs the bipartite double cover $\mathcal{H}^{\prime}$ of $\mathcal{H}$, finds a maximal matching in the bipartite graph $\mathcal{H}^{\prime}$, and maps the matching back to the original graph $\mathcal{H}$. Informally, the algorithm proceeds as follows:

- On odd rounds, each node sends proposals to its neighbours, in the order of increasing port numbers, until a proposal is accepted or the list of neighbours is exhausted.

- On even rounds, each node receives proposals and accepts the first proposal it gets (if any), breaking the ties with its port numbers.

Each edge with an accepted proposal is added to $P$. For a node $v$ of $\mathcal{H}$, there are at most two edges incident to $v$ in $P$ : an edge $\{v, u\}$ such that $v$ proposed and $u$ accepted, and another edge $\{v, t\}$ such that $t$ proposed and $v$ accepted.

Finally, the algorithm outputs the set $D=M \cup P$.

\subsection{Feasibility}

By construction, the set $D$ dominates all edges. To see this, let $\{v, u\} \in E_{\mathcal{G}}$ be an edge that is not dominated by $M$; hence it is part of the subgraph $\mathcal{H}$ that we consider in Phase III. If $v$ does not send a proposal to $u$, then $v$ must have received an acceptance earlier and $v$ is covered 
by $P$. Otherwise $u$ receives at least one proposal and hence becomes covered by $P$.

\subsection{Properties}

Let us then proceed to analyse the approximation factor. In the analysis, we need the following properties of $M$ and $P$.

(a) The sets $M$ and $P$ are node-disjoint, $M$ is a matching, and $P$ is a 2 -matching in $\mathcal{G}$.

(b) If $v \in V_{\mathcal{G}}$ has an odd degree, then $v$ is covered by $M$, or there is a neighbour $u$ of $v$ that is covered by $M$.

(c) If $\{v, u\} \in P$ then $d_{\mathcal{G}}(v)=d_{\mathcal{G}}(u)$.

The algorithm clearly preserves property (a). Property (b) follows from phase I. To verify property (c), observe that if $d_{\mathcal{G}}(v) \neq d_{\mathcal{G}}(u)$, we would have covered $v$ or $u$ in phase II.

\subsection{Definitions}

For a set $X \subseteq E_{\mathcal{G}}$ of edges, we say that a node $v \in V_{\mathcal{G}}$ is an $X$-node if it is covered by $X$. Hence each node is an $M$-node, a $P$-node, or neither.

By property (b) above, we can construct a set $C \subseteq E_{\mathcal{G}}$ of edges such that (i) each edge $e \in C$ joins a $P$-node and an $M$-node, and (ii) each $P$-node with an odd degree is incident to exactly one edge in $C$. Note that $M, P$, and $C$ are disjoint subsets of $E_{\mathcal{G}}$. Define $F=E_{\mathcal{G}} \backslash(M \cup P \cup C)$.

Now let $D^{*}$ be an arbitrary maximal matching in $\mathcal{G}$; in particular, $D^{*}$ can be a minimum maximal matching and hence a minimum edge dominating set for $\mathcal{G}$ (recall Section 1.1) We proceed to show that $|D|$ is not too large in comparison with $\left|D^{*}\right|$.

Each node covered by $D^{*}$ is called an internal node, and all other nodes are called external nodes; we are primarily interested in internal $P$-nodes (nodes covered by $P$ and $D^{*}$ ) and external $P$-nodes (nodes covered by $P$ but not $D^{*}$ ). Note that each edge is incident to at most one external node; otherwise $D^{*}$ would not be maximal.

\subsection{Costs}

We assign a cost $c(v)$ to each internal node $v$ as follows:

(i) Initially, $c(v) \leftarrow 0$ for all $v$.

(ii) For each $\{u, v\} \in D$ that connects an internal node $u$ and an external node $v$, we add $c(u) \leftarrow c(u)+1$.

(iii) For each $\{u, v\} \in D$ that connects two internal nodes $u$ and $v$, we add $c(u) \leftarrow c(u)+1 / 2$ and $c(v) \leftarrow c(v)+1 / 2$.

By construction, $2 c(v) \in\{0,1,2,3,4\}$, and the total cost of all internal nodes equals the size of the edge dominating set $D$. Let $I$ be the set of all internal nodes, and let $I_{x}=$ $|\{v \in I: c(v)=x / 2\}|$ be the number of internal nodes of cost $x / 2$. Observe that

$$
2\left|D^{*}\right|=|I|=\sum_{x} I_{x}, \quad 2|D|=\sum_{x} x I_{x} .
$$

If we had $c(v)=2$ for all $v \in I$, we would have $|D|=4\left|D^{*}\right|$. In what follows, we show that not all internal nodes can have $c(v)=2$.

\subsection{Weights}

We assign a weight $w(e)$ to each edge $e \in E_{\mathcal{G}}$ as follows:

(i) If $e=\{u, v\} \in F \cup C$ and $u$ is an external $P$-node, let $w(e)=2$.

(ii) If $e=\{u, v\} \in P$ and $u$ is an external $P$-node, let $w(e)=2-d_{\mathcal{G}}(u)$. (iii) Otherwise, $w(e)=0$.

Let $w(v)$ be the total weight of the edges incident to $v \in$ $V_{\mathcal{G}}$. Observe that each edge with a non-zero weight connects an external $P$-node to an internal node. Hence the total weight $w\left(E_{\mathcal{G}}\right)$ of all edges in the graph can be derived by two equivalent means: (i) summing $w(v)$ over all external $P$-nodes $v$, and (ii) summing $w(v)$ over all internal nodes $v$. We use this observation in a double-counting argument.

\subsection{Double Counting}

Let $v$ be an external $P$-node. There are at most 2 edges in $P$ that are incident to $v$, and hence at least $2 d_{\mathcal{G}}(v)-2$ edges in $F \cup C$ that are incident to $v$. Hence the total weight is $w(v) \geq 0$. In particular, the total weight of all edges in the graph is non-negative.

In the following, we consider an internal node $v$, and derive an upper bound on $w(v)$ as a function of $c(v)$.

If $c(v)=2$, then $v$ has to be incident to two edges in $D$, and these edges have to join $v$ and an external node. Hence the neighbours of $v$ can be classified as follows: there are two external nodes $s$ and $t$, with $\{v, s\} \in P,\{v, t\} \in P$; there is one internal node $u$, with $\{v, u\} \in D^{*}$, and finally $d_{\mathcal{G}}(v)-3$ other nodes $x_{1}, x_{2}, \ldots$, with $\left\{v, x_{i}\right\} \in F \cup C$. Вy property $(\mathrm{c})$, we have $d_{\mathcal{G}}(v)=d_{\mathcal{G}}(s)=d_{\mathcal{G}}(t)$. Hence the weights of the edges are $w(\{v, s\})=w(\{v, t\})=2-d_{\mathcal{G}}(v)$, $w(\{v, u\})=0$, and $w\left(\left\{v, x_{i}\right\}\right) \in\{0,2\}$ for each $i$, depending on whether $x_{i}$ happens to be an external $P$-node. It follows that the total weight of incident edges is

$$
w(v) \leq 2\left(2-d_{\mathcal{G}}(v)\right)+\left(d_{\mathcal{G}}(v)-3\right) 2=-2 .
$$

If $c(v)=3 / 2$, then $v$ has to have two neighbours, an external $P$-node $s$ and an internal $P$-node $t$ such that $\{v, s\} \in P$ and $\{v, t\} \in P$. Again, $d_{\mathcal{G}}(v)=d_{\mathcal{G}}(s)$, and hence we have assigned the weight $w(\{v, s\})=2-d_{\mathcal{G}}(v)$. There are two sub-cases. First, if $d_{\mathcal{G}}(v)=\Delta$, then $v$ is also adjacent to an $M$-node $u$ such that $\{v, u\} \in C$; by the choice of $w$, we have $w(\{v, u\})=0$. In addition to $s, t$, and $u$, there are $\Delta-3$ other nodes $x_{1}, x_{2}, \ldots$ adjacent to $v$; we have $\left\{v, x_{i}\right\} \in F$ and hence $w\left(\left\{v, x_{i}\right\}\right) \leq 2$. It follows that the total weight of incident edges is

$$
w(v) \leq 2-\Delta+(\Delta-3) 2<\Delta-3 .
$$

Otherwise $d_{\mathcal{G}}(v) \leq \Delta-1$, and $v$ is adjacent to $d_{\mathcal{G}}(v)-2$ other nodes $x_{1}, x_{2}$ in addition to $v$; we have $\left\{v, x_{i}\right\} \in F \cup C$ and hence $w\left(\left\{v, x_{i}\right\}\right) \leq 2$. It follows that the total weight is

$$
w(v) \leq 2-d_{\mathcal{G}}(v)+\left(d_{\mathcal{G}}(v)-2\right) 2 \leq \Delta-3 .
$$

If $c(v)=1$, we always have at least two edges incident to $v$ with a non-positive weight: If $v$ is incident to two edges in $D$, then we have two internal $P$-nodes $s$ and $t$ with $\{v, s\} \in P$ and $\{v, t\} \in P$; since each of $s, t$, and $u$ is internal, these edges have zero weight. Otherwise $v$ is incident to only one edge in $D$, let it be $\{v, s\} \in D$. In that case $s$ has to be an external node; hence there has to be another internal node $t$ with $\{v, t\} \in D^{*}$. The weight of $\{v, t\}$ is zero, as it joins a pair of internal nodes, and the weight of $\{v, s\}$ is $2-d_{\mathcal{G}}(v) \leq 0$ if $\{v, s\} \in P$ and zero if $\{v, s\} \in M$. We conclude that $v$ is incident to at most $d_{\mathcal{G}}(v)-2$ edges with a positive weight; since the weight of any edge is at most 2 , we have the upper bound

$$
w(v) \leq\left(d_{\mathcal{G}}(v)-2\right) 2 \leq 2 \Delta-4 .
$$


Finally, if $c(v) \leq 1 / 2$, it is sufficient to note that $v$ is adjacent to another internal node $u$ with $\{v, u\} \in D^{*}$, and $w(\{v, u\})=0$. Hence there are at most $d_{\mathcal{G}}(v)-1$ edges incident to $v$ with a positive weight; we have the upper bound

$$
w(v) \leq\left(d_{\mathcal{G}}(v)-1\right) 2 \leq 2 \Delta-2 .
$$

Summing over all internal nodes $i \in I$, we can derive the following upper bound on the total weight of all edges:

$W=-2 I_{4}+(\Delta-3) I_{3}+(2 \Delta-4) I_{2}+(2 \Delta-2) I_{1}+(2 \Delta-2) I_{0}$.

Since $0 \leq w\left(E_{\mathcal{G}}\right) \leq W$, we have $W \geq 0$ and

$$
2 I_{4} \leq(\Delta-3) I_{3}+(2 \Delta-4) I_{2}+(2 \Delta-2) I_{1}+(2 \Delta-2) I_{0} .
$$

\subsection{Approximation Ratio}

Now we are ready to derive an upper bound on the approximation ratio of the algorithm $\mathcal{A}(\Delta)$ :

$$
\begin{aligned}
\frac{|D|}{\left|D^{*}\right|} & =\frac{\sum_{x} x I_{x}}{\sum_{x} I_{x}}=\frac{4 I_{4}+3 I_{3}+2 I_{2}+I_{1}}{I_{4}+I_{3}+I_{2}+I_{1}+I_{0}} \\
& =4-\frac{2 I_{3}+4 I_{2}+6 I_{1}+8 I_{0}}{2 I_{4}+2 I_{3}+2 I_{2}+2 I_{1}+2 I_{0}} \\
& \leq 4-\frac{2 I_{3}+4 I_{2}+6 I_{1}+8 I_{0}}{(\Delta-1) I_{3}+(2 \Delta-2) I_{2}+2 \Delta I_{1}+2 \Delta I_{0}} \\
& \leq 4-\frac{2}{\Delta-1}=4-\frac{1}{k} ;
\end{aligned}
$$

here we have used the assumption that $\Delta \geq 3$ and hence $3 / \Delta \geq 2 /(\Delta-1)$. We conclude that $\alpha(\Delta) \leq 4-1 / k$, and Theorem 5 follows.

\section{ACKNOWLEDGEMENTS}

Thanks to Matti Åstrand, Patrik Floréen, Petteri Kaski, Topi Musto, Valentin Polishchuk, Joel Rybicki, and Jara Uitto for discussions, and to anonymous reviewers for their comments. This work was supported in part by the Academy of Finland, Grant 132380.

\section{REFERENCES}

[1] R. B. Allan and R. Laskar. On domination and independent domination numbers of a graph. Discrete Math., 23(2):73-76, 1978.

[2] D. Angluin. Local and global properties in networks of processors. In Proc. 12th Symposium on Theory of Computing (STOC 1980), pages 82-93. ACM Press, 1980.

[3] M. Åstrand, P. Floréen, V. Polishchuk, J. Rybicki, J. Suomela, and J. Uitto. A local 2-approximation algorithm for the vertex cover problem. In Proc. 23rd Symposium on Distributed Computing (DISC 2009), volume 5805 of $L N C S$, pages 191-205. Springer, 2009.

[4] M. Astrand and J. Suomela. Fast distributed approximation algorithms for vertex cover and set cover in anonymous networks. In Proc. 22nd Symposium on Parallelism in Algorithms and Architectures (SPAA 2010). ACM Press, 2010.

[5] H. Attiya, M. Snir, and M. K. Warmuth. Computing on an anonymous ring. J. ACM, 35(4):845-875, 1988.

[6] B. S. Baker. Approximation algorithms for NP-complete problems on planar graphs. J. ACM, 41(1):153-180, 1994.
[7] P. Boldi and S. Vigna. An effective characterization of computability in anonymous networks. In Proc. 15th Symposium on Distributed Computing (DISC 2001), volume 2180 of $L N C S$, pages 33-47. Springer, 2001.

[8] R. Carr, T. Fujito, G. Konjevod, and O. Parekh. A $2 \frac{1}{10}$-approximation algorithm for a generalization of the weighted edge-dominating set problem. J. Comb. Optim., 5(3):317-326, 2001.

[9] M. Chlebík and J. Chlebíková. Approximation hardness of edge dominating set problems. J. Comb. Optim., 11(3):279-290, 2006.

[10] A. Czygrinow, M. Hańćkowiak, and W. Wawrzyniak. Fast distributed approximations in planar graphs. In Proc. 22nd Symposium on Distributed Computing (DISC 2008), volume 5218 of LNCS, pages 78-92. Springer, 2008.

[11] R. Diestel. Graph Theory. Springer, 3rd edition, 2005.

[12] T. Fujito and H. Nagamochi. A 2-approximation algorithm for the minimum weight edge dominating set problem. Discrete Appl. Math., 118(3):199-207, 2002.

[13] M. Hańćkowiak, M. Karoński, and A. Panconesi. On the distributed complexity of computing maximal matchings. In Proc. 9th Symposium on Discrete Algorithms (SODA 1998), pages 219-225. SIAM, 1998.

[14] M. Hańćkowiak, M. Karoński, and A. Panconesi. On the distributed complexity of computing maximal matchings. SIAM J. Discrete Math., 15(1):41-57, 2001.

[15] H. B. Hunt III, M. V. Marathe, V. Radhakrishnan, S. S. Ravi, D. J. Rosenkrantz, and R. E. Stearns. NC-approximation schemes for NP- and PSPACE-hard problems for geometric graphs. $J$. Algorithms, 26(2):238-274, 1998.

[16] D. R. Kowalski and A. Malinowski. How to meet in anonymous network. Theoret. Comput. Sci., 399(1-2):141-156, 2008.

[17] C. Lenzen and R. Wattenhofer. Leveraging Linial's locality limit. In Proc. 22nd Symposium on Distributed Computing (DISC 2008), volume 5218 of LNCS, pages 394-407. Springer, 2008.

[18] M. Naor and L. Stockmeyer. What can be computed locally? SIAM J. Comput., 24(6):1259-1277, 1995.

[19] A. Panconesi and R. Rizzi. Some simple distributed algorithms for sparse networks. Distributed Computing, 14(2):97-100, 2001.

[20] J. Petersen. Die Theorie der regulären graphs. Acta Mathematica, 15(1):193-220, 1891.

[21] V. Polishchuk and J. Suomela. A simple local 3-approximation algorithm for vertex cover. Inform. Process. Lett., 109(12):642-645, 2009.

[22] J. Suomela. Survey of local algorithms, 2009. Manuscript submitted for publication.

[23] M. Yamashita and T. Kameda. Computing functions on asynchronous anonymous networks. Mathematical Systems Theory, 29(4):331-356, 1996.

[24] M. Yamashita and T. Kameda. Computing on anonymous networks: Part I - characterizing the solvable cases. IEEE Trans. Parallel Distrib. Systems, $7(1): 69-89,1996$.

[25] M. Yannakakis and F. Gavril. Edge dominating sets in graphs. SIAM J. Appl. Math., 38(3):364-372, 1980. 